\title{
Simvastatin Protects Dopaminergic Neurons Against MPP+-Induced Oxidative Stress and Regulates the Endogenous Anti- Oxidant System Through ERK
}

\author{
Junqiang Yan ${ }^{\mathrm{a}, \mathrm{b}}$ Liang Qiao ${ }^{\mathrm{b}}$ Jiannan Wu $\mathrm{Hua}^{\mathrm{a}}$ Fan $^{\mathrm{c}}$ Jiachun Sun ${ }^{\mathrm{d}}$ \\ Yude Zhanga
}

\begin{abstract}
aNeurological Diseases Institute, The First Affiliated Hospital, College of Clinical Medicine of Henan University of Science and Technology, Luoyang, 'Department of Neurology, The First Affiliated Hospital, College of Clinical Medicine of Henan University of Science and Technology, Henan, 'Department of Pharmacy, The First Affliated Hospital, College of Clinical Medicine of Henan University of Science and Technology, Luoyang, dDepartment of Oncology, The First Affiliated Hospital, College of Clinical Medicine of Henan University of Science and Technology, Luoyang, China
\end{abstract}

\author{
Key Words \\ Simvastatin • Parkinson's disease $・$ ERK1/2 • NOX2 • Anti-oxidant
}

\begin{abstract}
Background/Aims: Many clinical studies have demonstrated that statins, especially simvastatin, can decrease the incidence of Parkinson's disease (PD). However, the specific underlying mechanism remains unclear. This study aimed to investigate how simvastatin affects experimental parkinsonian models via the regulation of extracellular signal-regulated kinase 1/2 (ERK1/2)-mediated activation of the anti-oxidant system. Methods: I-Methyl-4phenylpyridine ion $\left(\mathrm{MPP}^{+}\right)$-treated $\mathrm{SH}-\mathrm{SY} 5 \mathrm{Y}$ cells and substantia nigra neurons were used to investigate the neuroprotective effect of simvastatin. After incubation with $\mathrm{MPP}^{+}$and/or simvastatin for $24 \mathrm{~h}$, the MTT assay was used to assess cell viability. Reactive oxygen species (ROS) levels were measured using $2^{\prime}, 7^{\prime}$-dichlorofluorescin diacetate, while cellular superoxide dismutase (SOD) levels were determined based on the blue formazan produced by the reduction of nitroblue tetrazolium. The level of cellular grade micro-reduced glutathione (GSH) was measured with 5,5'-dithiobis-(2-nitrobenzoic acid). Meanwhile, the malondialdehyde content released from $\mathrm{SH}-\mathrm{SY} 5 \mathrm{Y}$ cells and substantia nigra neuronal cells exposed to different culture media was calculated based on the condensation reaction involving thiobarbituric acid. The mRNA levels of genes encoding nuclear factor (erythroid-derived 2)-like 2 (Nrf2), heme oxygenase $1(\mathrm{HO}-1)$, and $\mathrm{NAD}(\mathrm{P}) \mathrm{H}$ dehydrogenase (quinone) 1 (NQO-1) were determined by a quantitative polymerase chain reaction assay, while the ERK, Nrf2, HO-1, NOX2, and NQO-1 protein levels were analyzed by western blot. Additionally, ERK small interfering RNA (siRNA)
\end{abstract}

Prof. Dr. Junqiang Yan, $\mathrm{MD}, \mathrm{PhD}$
Neurological Diseases Institute, The First Affiliated Hospital of Henan University of Science and Technology, Jinghua Road 24, Luoyang, Henan 471003 (China) Tel. +86-379-64830495, E-Mail yanjq20062007@126.com 


\section{Cellular Physiology Cell Physiol Biochem 2018;51:1957-1968

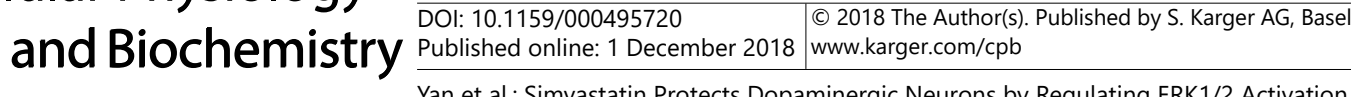 \\ of Anti-Oxidants}

was used to investigate the mechanisms underlying $\mathrm{MPP}^{+}$-induced oxidative stress and the regulation of the endogenous anti-oxidant system. Results: Simvastatin (1.5 $\mu \mathrm{M})$ enhanced the viability of SH-SY5Y cells and primary neurons treated with $\mathrm{MPP}^{+}$, and significantly alleviated the oxidative stress induced by MPP+ in SH-SY5Y cells by regulating the production of SOD, analytical grade micro-reduced GSH, and ROS, which may be associated with the activation of the Nrf2 anti-oxidant system. An analysis involving ERK1/2 siRNA revealed that simvastatin can inhibit NOX2 expression via the activation of ERK1/2 in the MPP+-treated PD cell model. Conclusion: Our results provide strong evidence that ERK1/2-mediated modulation of the anti-oxidant system after simvastatin treatment may partially explain the anti-oxidant activity in experimental parkinsonian models. These findings contribute to a better understanding of the critical roles of simvastatin via the ERK1/2-mediated modulation of the anti-oxidant system, which may be relevant for treating PD.

\section{Introduction}

Parkinson's disease (PD) is a neurodegenerative disorder characterized by the loss of dopaminergic neurons in the substantia nigra and by the formation of Lewy bodies [1]. Additionally, PD is accompanied by neuronal oxidative stress injury [2]. The classic PD cell model generally uses 1-methyl-4-phenyl-pyridinium (MPP+), rotenone, or 6-hydroxydopamine (6-OHDA) as neurotoxins that induce varying degrees of oxidative stress injury [3]. In the $\mathrm{MPP}^{+}$-induced $\mathrm{PD}$ cell model, $\mathrm{MPP}^{+}$can inactivate tyrosine hydroxylase in dopaminergic neurons and inhibit the oxidative stress injury of mitochondrial complex I [4].

Statins inhibit 5-hydroxy-3-methylglutaryl-coenzyme A (HMG-CoA) reductase, which plays a key role in the production of cholesterol. Furthermore, statins belong to a class of lipidlowering drugs widely used in the treatment of hyperlipidemia. Based on neurodegenerative disease research, different statins may have a beneficial effect in the treatment of dementia [5], PD [6], and Alzheimer's disease [7].

Our previous study on simvastatin (Sim) therapy involving patients with PD confirmed that Sim is able to modulate NMDA receptors [8] and protect against dopaminergic neurodegeneration through the PI3K/Akt/caspase 3 pathway [9]. In a related study, we observed that the anti-oxidative stress function of Sim in dopaminergic neurons is very prominent. Previous evidence suggests that Sim is able to activate Nrf2 and respond positively to oxidative stress caused by $\mathrm{H}_{2} \mathrm{O}_{2}[10]$. We have determined the anti-oxidant properties of Sim. Moreover, we conducted an in-depth study to investigate its effects and the mechanism underlying the oxidative stress induced by $\mathrm{MPP}^{+}$in dopaminergic neuronal cells.

\section{Materials and Methods}

\section{Cell culture and treatment}

SH-SY5Y cells, which were obtained from Sun Ye San University (Guangzhou, China), were grown in Dulbecco's modified Eagle's medium (DMEM; HyClone, Logan, UT) with 10\% fetal bovine serum (Gibco, Gaithersburg, MD) and 1\% penicillin-streptomycin.The experiment involving substantia nigra neurons was conducted as previously described [11]. Newborn C57BL/6J mice were obtained within $24 \mathrm{~h}$ of birth from HFK Bioscience Co., Ltd. (Beijing, China). Isolated neurons were cultured in DMEM containing F-12 and 1 $\mathrm{mM}$ glutamine. The SH-SY5Y cells and primary culture substantia nigra neurons were maintained at $37^{\circ} \mathrm{C}$ in an incubator containing 5\% carbon dioxide. Sim was prepared with an ethanol-sodium hydroxide solution, stored at $-20^{\circ} \mathrm{C}$, and diluted to specific concentrations in the culture medium.

To produce oxidative stress, $100 \mathrm{mM} \mathrm{MPP}^{+}$(M7068, Sigma-Aldrich, St. Louis, M0) was freshly prepared in deionized water as a stock solution prior to each experiment. The SH-SY5Y cells and substantia nigra neurons were incubated with specific concentrations of Sim and $\mathrm{MPP}^{+}(1 \mathrm{mM})$. 


\section{Cellular Physiology Cell Physiol Biochem 2018;51:1957-1968 \begin{tabular}{l|l|l} 
and Biochemistry Published online: 1 December 2018 & $\begin{array}{l}\text { (c) } 2018 \text { The Author(s). Published by S. Karger AG, Basel } \\
\text { www.karger.com/cpb }\end{array}$ \\
\hline
\end{tabular} \\ Yan et al.: Simvastatin Protects Dopaminergic Neurons by Regulating ERK1/2 Activation of Anti-Oxidants}

\section{Cell viability}

Cell viability was determined based on the MTT assay (Solarbio, Beijing, China). The SH-SY5Y cells and substantia nigra neurons were treated with Sim or MPP', after which $0.5 \mathrm{mg} / \mathrm{ml}$ MTT solution was added to the culture medium, which was then incubated for $4 \mathrm{~h}$. After adding dimethyl sulfoxide to the cells, the absorbance at $570 \mathrm{~nm}$ was measured with an EnSpire 2300 multimode plate reader (Perkin Elmer, Singapore). The survival rate of the control group was $100 \%$, and the survival rates of the other groups were comparable with that of the control group.

\section{Malondialdehyde assay}

The SH-SY5Y cells were treated with Sim or MPP ${ }^{+}$and were centrifuged at $12,000 \times \mathrm{g}$ for $5 \mathrm{~min}$ at $4^{\circ} \mathrm{C}$ after cells were lysed. According to the manufacturer's instructions, the malondialdehyde (MDA) level of the supernatant was measured based on the difference between the absorbances at $532 \mathrm{~nm}$ and $600 \mathrm{~nm}$, which were determined with a multimode plate reader.

\section{Glutathione assay}

The glutathione (GSH) content was measured according to the manufacturer's recommendations for the glutathione assay kit (Solarbio). The GSH levels were quantified based on the absorbance at $412 \mathrm{~nm}$, which was determined with a multimode plate reader.

\section{Reactive oxygen species assay}

Black 96-well plates were inoculated with cells. After a 24-h incubation, $10 \mu \mathrm{M} \mathrm{2} 2^{\prime}, 7^{\prime}$-dichlorofluorescin diacetate was added. The cells were incubated at $37^{\circ} \mathrm{C}$ for $30 \mathrm{~min}$ and washed twice with DMEM before being treated with $\mathrm{MPP}^{+}$and Sim for $24 \mathrm{~min}$. After $48 \mathrm{~h}$, the absorbance at $488 \mathrm{~nm}$ was determined with a multimode plate reader.

\section{Superoxide dismutase activity assay}

The SH-SY5Y cells were treated with Sim or MPP ${ }^{+}$and were centrifuged at $12,000 \times \mathrm{g}$ for $5 \mathrm{~min}$ at $4^{\circ} \mathrm{C}$ after cells were lysed. According to the manufacturer's instructions, the superoxide dismutase (SOD) level of the supernatant was measured based on the absorbance at $560 \mathrm{~nm}$, which was determined with a multimode plate reader.

\section{Quantitative polymerase chain reaction assay}

Total RNA was extracted from the SH-SY5Y cells and substantia nigra neurons using the Trizol reagent as per the manufacturer's protocol. Total RNA $(2 \mu \mathrm{g})$ was used as the template for a reverse transcription to cDNA with the HiFi-Script cDNA synthesis kit. The quantitative polymerase chain reaction (qPCR) assay was completed with the ABI 7500 system, SYBR Green I, and the following primers:

Nrf2 forward primer (5'-TCTCGGATCACTCTCTGGCA-3');

reverse primer (5'-GTTACGAGGGGCCAACTCC-3')

HO-1 forward primer ( $5^{\prime}$-GGCCTCCCTGTACCACATCTA-3');

reverse primer (5'-GGAAGTAGACAGGGGCGAAGA-3')

NQO-1 forward primer (5'-TGCAGCGGCTTTGAAGAAG-3');

reverse primer (5'-AAGTTCGCAGGGTCCTTCAG-3')

GAPDH forward primer (5'-CTCCGGGTGATGCTTTTCCTA-3');

reverse primer (5'-AGTTGAGGTCAATGAAGGGGTC-3')

Each 20- $\mu \mathrm{l} \mathrm{qPCR}$ solution comprised $2 \mu \mathrm{l}$ target cDNA. The PCR program was as follows: $95^{\circ} \mathrm{C}$ for $5 \mathrm{~min}$; 35 cycles at $95^{\circ} \mathrm{C}$ for $15 \mathrm{~s}, 65^{\circ} \mathrm{C}$ for $15 \mathrm{~s}$, and $72^{\circ} \mathrm{C}$ for $30 \mathrm{~s}$. Each reaction was repeated three times. For the negative control reaction, cDNA was replaced with an equal volume of $\mathrm{ddH}_{2} \mathrm{O}$. The target gene expression levels were calculated according to the relative quantitative $2^{-\Delta c t}$ method [11].

\section{Western blot assay}

The SH-SY5Y cells were lysed and centrifuged, after which the protein in the supernatant was quantified using bicinchoninic acid. Nucleoproteins were extracted with the Nucleoprotein Extraction Kit (Sangon Biotech, Shanghai, China), following the manufacturer's instructions. Total protein $(20 \mu \mathrm{g})$ was separated by $10 \%$ sodium dodecyl sulfate polyacrylamide gel electrophoresis and then transferred to 0.22 - 


\section{Cellular Physiology Cell Physiol Biochem 2018;51:1957-1968 \\ \begin{tabular}{ll|l} 
and Biochemistry & $\begin{array}{l}\text { DOI: } 10.1159 / 000495720 \\
\text { Published online: } 1 \text { December } 2018\end{array}$ & $\begin{array}{l}\text { @ } 2018 \text { The Author(s). Published by S. Karger AG, Basel } \\
\text { www.karger.com/cpb }\end{array}$ \\
\cline { 2 - 3 }
\end{tabular}}

of Anti-Oxidants

$\mu$ m polyvinylidene difluoride membranes. The membranes were blocked with $5 \%$ skim milk diluted in a Tris-buffered saline solution containing 1\% Tween-20 (TBST). The membranes were incubated overnight at $4^{\circ} \mathrm{C}$ in solutions with primary antibodies (1:1000) against one of Nrf2, HO-1, NQO-1, Histone H3 (Abcam, Cambridge, UK), NOX2, Erk1/2, or Phospho ERK1/2 (Cell Signaling Technology, Danvers, MA) or primary antibodies (1:5000) against GAPDH (CWBio, Beijing, China). The membranes were washed three times with TBST before an incubation in solutions with the corresponding secondary antibody (CWBio) to detect proteins. All experiments were conducted at least three times. Image J was used to detect the density of the visualized proteins relative to that of the loading control.

\section{Immunofluorescence}

The SH-SY5Y cells were seeded on lysine-treated circular slides in plates with 12 holes and immobilized with polyoxymethylene. The samples were blocked with $10 \%$ goat serum (Solarbio) and incubated in a $1 \%$ bovine serum albumin solution containing a primary antibody (1:200) against Nrf2. The samples were then incubated with the Alexa Fluor 555 (Thermo Fisher Scientific, Waltham, MA) secondary antibody (1:1000). Slides were sealed after adding DAPI and then observed with an LSM 780 confocal microscope (Zeiss, LSM 780).

Small interfering RNA silencing

The SH-SY5Y cells were treated with $N r f 2$ and ERK1/2 small interfering RNAs (siRNAs) (Santa Cruz

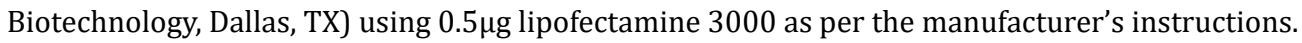

Nicotinamide adenine dinucleotide phosphate oxidase activity assay

The activation of NADH oxidase (NOX) was analyzed in accordance with the instructions of the Micro NADH Oxidase (NOX) assay kit(BC0635, Solarbio, Beijing, China). The degree of NOX activation was based on the reduction of 2, 6-dichlorophenol indigo, which was measured with a spectrophotometer $(600 \mathrm{~nm}$ wavelength). The NOX activity rate was recorded relative to that of the control group.

\section{Statistical analysis}

Data were expressed as the mean \pm standard error of the mean. Comparisons between two groups were analyzed using an unpaired two-sided Student's $t$-test. Analysis of variance and Tukey's post hoc analysis were used to evaluate differences among the various treatments (SPSS version 17.0, SPSS Inc., Chicago, IL), with $p<0.05$ considered statistically significant for all analyses.

\section{Results}

Sim protects SH-SY5Y cells and primary neurons against MPP+-induced cytotoxicity

As shown in Fig. 1a, the cytotoxicity of MPP+-treated cells was determined according to the MTT method. The survival rates of SH-SY5Y cells after a 24-h incubation in $0.1,0.25,0.5$, or $1 \mathrm{mM} \mathrm{MPP}{ }^{+}$were compared with that of the control group $\left({ }^{*} p<0.05\right.$ or $\left.{ }^{* * *} p<0.001, \mathrm{n}=5\right)$.

We observed that there were no significant differences among the SH-SY5Y cells exposed to $0.1,0.5,1.0,1.5,2.5$, or $5.0 \mu \mathrm{M}$ Sim for $24 \mathrm{~h}$ (Fig. $1 \mathrm{~b}$ ). However, greater cytotoxicity was observed for SH-SY5Y cells treated with a high dose $(5 \mu \mathrm{M})$ of Sim over an extended period (72 h) than for the control cells (Fig. $1 \mathrm{c})\left({ }^{*} p<0.05\right.$ or $\left.{ }^{* * *} p<0.001, \mathrm{n}=5\right)$.

The protective effect of Sim against $\mathrm{MPP}^{+}(1 \mathrm{mM})$-induced oxidative stress in SH-SY5Y cells and primary cultured substantia nigra neurons (PD model) was further investigated. As revealed in Fig. 1d, $\mathrm{MPP}^{+}$significantly decreased the viability of SH-SY5Y cells by 57.01 $\pm 2.60 \%\left({ }^{* * *} p<0.001, \mathrm{n}=5\right)$ and primary neurons by $47.14 \pm 2.80 \%\left({ }^{* * *} p<0.001, \mathrm{n}=5\right)$ compared with the control levels.

Finally, we analyzed the effect of Sim on cell survival and neuroprotection in $\mathrm{MPP}^{+}-$ induced oxidative stress PD cell models. As shown in Fig. 1e, $1.5 \mu \mathrm{M}$ Sim enhanced the viability of SH-SY5Y cells by $88.84 \pm 2.82 \%$ and primary neurons by $79.06 \pm 2.08 \%{ }^{\# \#} p<$ 0.01 or $^{\# \# \# ~} p<0.001, \mathrm{n}=5$ ) versus $\mathrm{MPP}^{+}$-treated cells. 


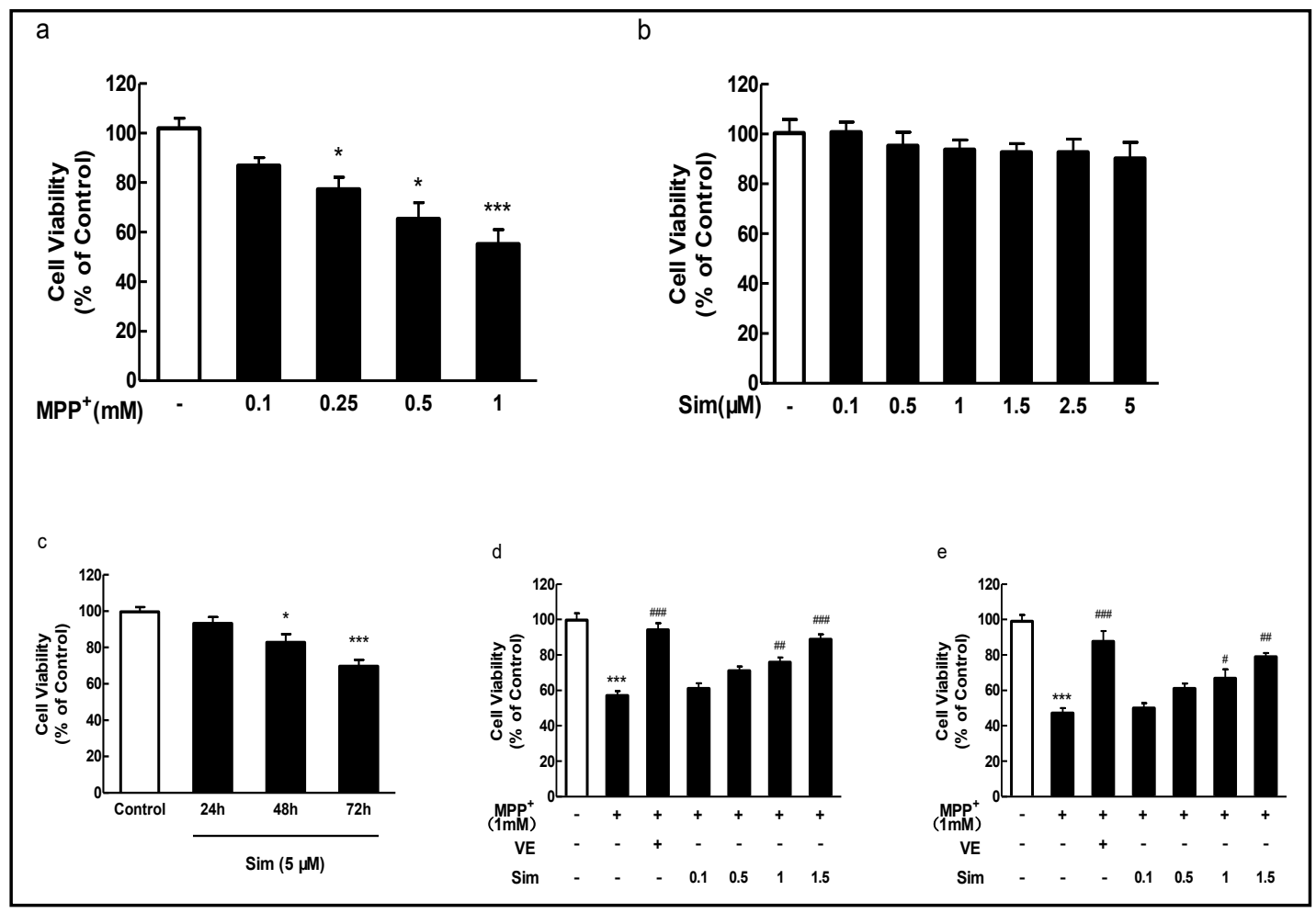

Fig. 1. Sim-protected neuronal cells against $\mathrm{MPP}^{+}$-induced cytotoxicity. (a) The cytotoxicity of SH-SY5Y cells incubated with $0.1,0.25,0.5$, or $1.0 \mathrm{mM} \mathrm{MPP}^{+}$was analyzed according to the MTT method. (b) The cytotoxicity of SH-SY5Y cells incubated with $0.1,0.5,1.0,1.5,2.5$, or $5.0 \mu \mathrm{M}$ Sim was analyzed according to the MTT method. (c) The cytotoxicity of SH-SY5Y cells incubated with $5 \mu \mathrm{M}$ Sim for 24, 48, and $72 \mathrm{~h}$ was analyzed according to the MTT method. (d-e) The cytotoxicities of SH-SY5Y cells (d) and primary cultured nigral neurons (e) treated with or without $0.1,0.5,1.0,1.5,2.5$, or $5.0 \mu \mathrm{M}$ Sim and $1 \mathrm{mM} \mathrm{MPP}^{+}$were analyzed according to the MTT method. The results of the control group are expressed as ${ }^{*} \mathrm{p}<0.05$ or ${ }^{* * *} \mathrm{p}<0.001$, and the results of the $\mathrm{MPP}^{+}$-treated group are expressed as ${ }^{\# \#} \mathrm{p}<0.01$ or $\# \#$ p $<0.001$.

Sim treatment may significantly alleviate oxidative stress induced by $\mathrm{MPP}^{+}$in $\mathrm{SH}-\mathrm{SY} 5 \mathrm{Y}$ cells

Oxidative stress affects the cytotoxicity of $\mathrm{MPP}^{+}$in the PD cell model. Therefore, the effect of Sim on $\mathrm{MPP}^{+}$-induced cytotoxicity was investigated by detecting the reactive oxygen species (ROS) and MDA levels, GSH content, and SOD activity. The ROS level significantly increased to $1.39 \pm 0.10$ in $\mathrm{MPP}^{+}$-treated SH-SY5Y cells, while it was only $1.03 \pm 0.07$ in the control cells $\left({ }^{*} p<0.05, \mathrm{n}=5\right.$ ) (Fig. 2a). Additionally, $1.5 \mu \mathrm{M}$ Sim significantly decreased the ROS level of MPP+-treated cells to $1.05 \pm 0.10 \%$ (Fig. 2 a) $\left({ }^{\#} p<0.05, \mathrm{n}=5\right.$ ).

MDA is a common marker of oxidative stress and oxygen toxicity because it is formed by the degradation of unsaturated lipids with ROS. The MDA content was significantly increased to $1.959 \pm 0.14 \mathrm{nmol} / \mathrm{mg}$ in the $\mathrm{MPP}^{+}$-treated group, but was only $0.69 \pm 0.09 \mathrm{nmol} / \mathrm{mg}$ in the control group (Fig. 2b). After treatments with 1.0 and $1.5 \mu \mathrm{M}$ Sim, the MDA level decreased significantly in cells exhibiting $\mathrm{MPP}^{+}$-induced oxidative stress to $1.474 \pm 0.19 \mathrm{nmol} / \mathrm{mg}\left({ }^{\#} p\right.$ $<0.05 \mathrm{n}=5)$ and $1.284 \pm 0.13 \mathrm{nmol} / \mathrm{mg}\left({ }^{\# \#} p<0.01, \mathrm{n}=5\right)$, respectively. Additionally, GSH, which is an important anti-oxidant that can be reduced by NADPH reductase, is another indicator of intracellular oxidative stress. Important anti-oxidants, such as SOD, exist in almost all living cells requiring oxygen.

As shown in Fig. 2c and d, compared with the corresponding control group, the GSH and SOD levels in SH-SY5Y cells significantly decreased after a treatment with MPP ${ }^{+}$from $6.06 \pm 0.45 \mu \mathrm{mol} / \mathrm{mg}$ to $2.87 \pm 0.31 \mu \mathrm{mol} / \mathrm{mg}$ and $30.97 \pm 2.57 \mathrm{U} / \mathrm{mg}$ to $11.76 \pm 1.52 \mathrm{U} / \mathrm{mg}$, 
Yan et al.: Simvastatin Protects Dopaminergic Neurons by Regulating ERK1/2 Activation of Anti-Oxidants

Fig. 2. Sim significantly decreased the oxidative stress induced by $\mathrm{MPP}^{+}$ in SH-SY5Y cells. (a-d) The ROS (a), MDA (b), GSH (c), and SOD (d) levels were measured in SH-SY5Y cells treated with or without 1.0 and 1.5 $\mu \mathrm{M}$ Sim and $1 \mathrm{mM}$ $\mathrm{MPP}^{+}$. The results of the control group are expressed as ${ }^{*} \mathrm{p}<0.05, \quad{ }^{* *} \mathrm{p}<0.01$, or ${ }^{* * *} \mathrm{p}<0.001$, and the results of the M P $\mathrm{P}^{+}-\mathrm{tr}$ e a te d
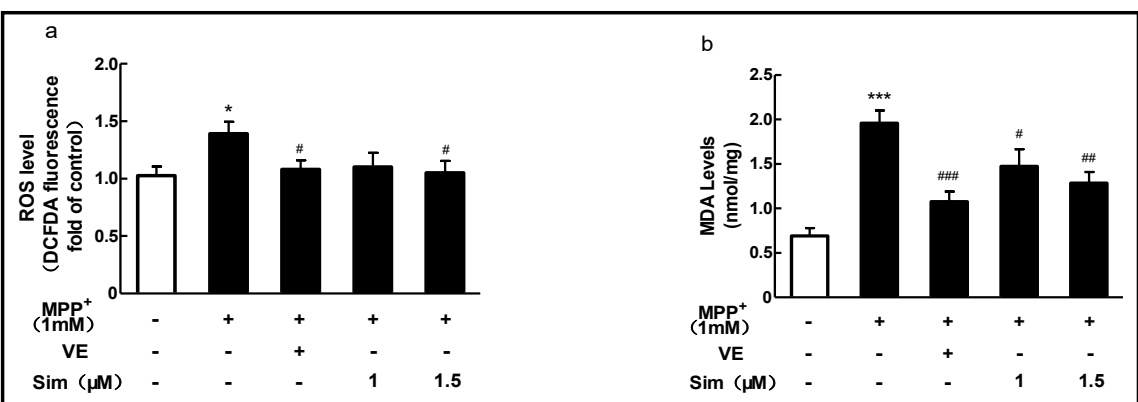

c
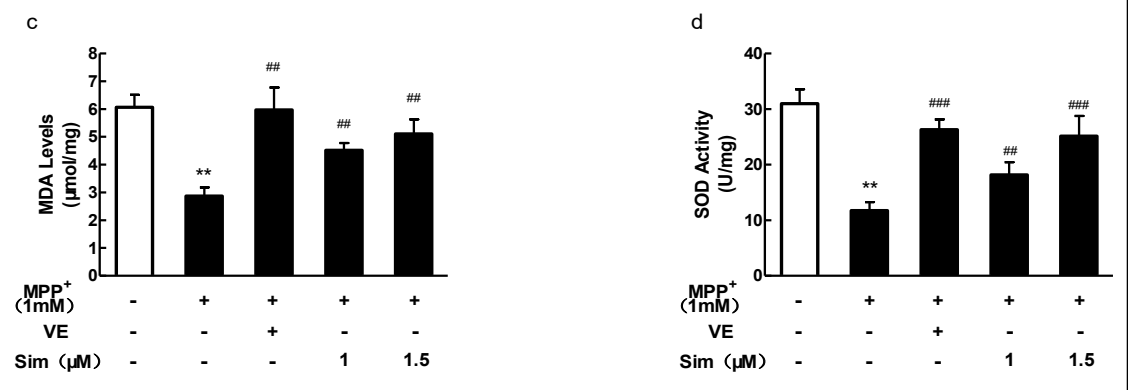

group are expressed as ${ }^{\# \#} \mathrm{p}<0.01$ or ${ }^{\# \# \#} \mathrm{p}<0.001$.

respectively $\left({ }^{* *} p<0.01, \mathrm{n}=5\right)$. Moreover, GSH and SOD levels in MPP+-treated SH-SY5Y cells exposed to 1.0 and $1.5 \mu \mathrm{M}$ Sim decreased in a dose-dependent manner ${ }^{\# \#} p<0.01$ or \#\#\# $p<$ $0.001, \mathrm{n}=5$ ).

Thus, these results suggested that Sim can protect SH-SY5Y cells by resisting the effects of oxidative stress induced by $\mathrm{MPP}^{+}$.

Sim mediated its protective effect by increasing the expression of the anti-oxidant-encoding NRF2 in a dose-dependent manner

The anti-oxidant effect of Nrf2 is considered to be the most likely factor responsible for the intracellular regulation of reduction and oxidation reactions. We hypothesized that a Sim treatment can increase the expression of anti-oxidant-encoding genes, such as Nrf2, in

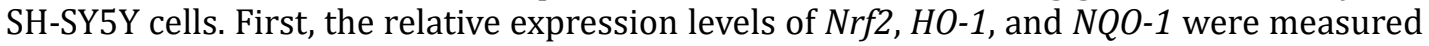
by qPCR. The mRNA levels of Nrf2 (Fig. 3a) $\left({ }^{*} p<0.05,{ }^{* *} p<0.01\right.$, or $\left.{ }^{* * *} p<0.001, \mathrm{n}=5\right), H O-1$ (Fig. 3b) $\left.{ }^{* * *} p<0.001, \mathrm{n}=5\right)$, and NQO-1 (Fig. 3c) $\left({ }^{* *} p<0.01\right.$ or ${ }^{* * *} p<0.001, \mathrm{n}=5$ ) after a $24-\mathrm{h}$ treatment with $0.1,0.5,1.0$, and $5.0 \mu \mathrm{M}$ Sim were significantly higher than the corresponding mRNA level in the control group, and were positively correlated with the Sim dose. Next, we observed the effect of Sim on Nrf2-related anti-oxidant system protein expression. The results showed that the Nrf2, HO-1, and NQO- 1 levels $\left({ }^{*} p<0.05,{ }^{* *} p<0.01\right.$, or ${ }^{* * *} p<0.001$, $\mathrm{n}=5$ ) were significantly higher in the treated cells than in the control cells (Fig. $3 \mathrm{~d}$ ). The relative values in Fig. 3e, f, and g increased to $240.20 \pm 22.15 \%, 167.80 \pm 20.77 \%$, and 183.20 $\pm 17.03 \%$, respectively. To confirm the effect of Sim on Nrf2 and determine whether it plays an important neuroprotective role, we analyzed Sim effects on the survival of the control and Nrf2-silenced SH-SY5Y cells exposed to $\mathrm{MPP}^{+}$. The nuclear Nrf2 levels in the control group and Sim treatment group are presented in Fig. 3h. After the addition of $1.5 \mu \mathrm{M}$ Sim, the nuclear Nrf2 level significantly increased (Fig. 3i) $\left({ }^{* * *} p<0.001, \mathrm{n}=5\right)$. Moreover, as indicated in Fig. $3 \mathrm{j}$, Nrf2 appeared in the cytoplasm after the $1.5 \mu \mathrm{M}$ Sim treatment. When Nrf2 expression was silenced, the protective effects of Sim appeared to be largely eliminated (Fig. 3k) (n = 5, $p<0.05$ vs. $\mathrm{MPP}^{+}+$scrambled-siRNA). 


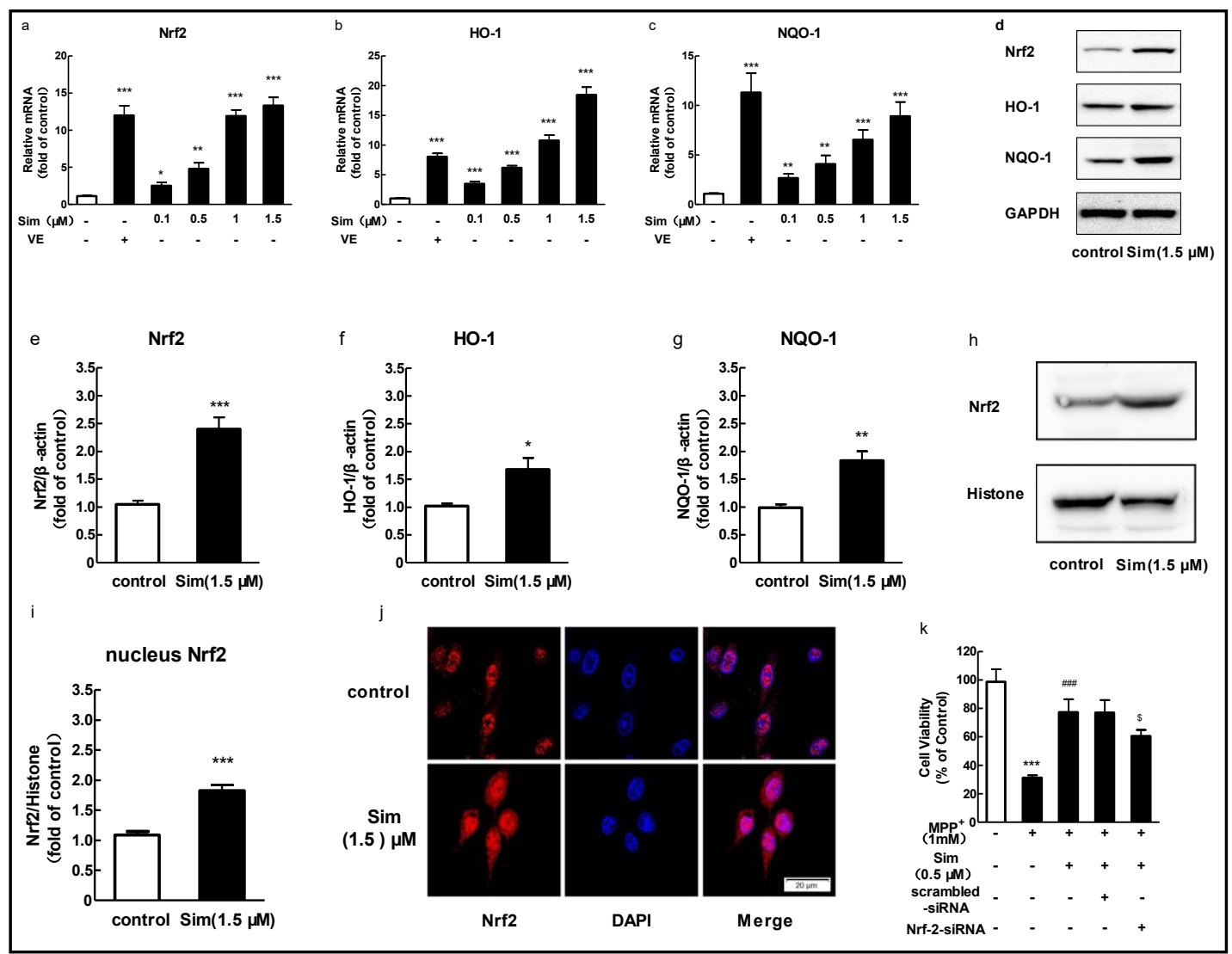

Fig. 3. Sim mediated its protective effect by modifying the expression of Nrf2-related anti-oxidant genes in a dose-dependent manner. (a-c) Fluorescent qPCR was used to analyze SH-SY5Y cells treated with 0.1, 0.5, 1.0, or $1.5 \mu \mathrm{M}$ Sim regarding the expression of Nrf2 (a), HO-1 (b), and NQO-1 (c). Vitamin E (VE) was used as a positive anti-oxidant control. (d) Western blot was used to examine SH-SY5Y cells treated with $1.5 \mu \mathrm{M}$ Sim regarding Nrf2 (e), HO-1 (f), and NQO-1 (g) protein expression levels. (h and i) Western blot was used to analyze SH-SY5Y cells treated with $1.5 \mu$ M Sim regarding nuclear Nrf2 levels. (j) Nrf2 immunofluorescence in SH-SY5Y cells (Red: Nrf2, Blue: DAPI). (k) Transfection with the target siRNA to inhibit Nrf2 expression in SH-SY5Y cells treated with $1 \mathrm{mM} \mathrm{MPP}$. The protective effect of Sim after silencing Nrf2 expression was detected according to the MTT method. Compared with the control group expressed as ${ }^{*} \mathrm{p}<0.05,{ }^{* *} \mathrm{p}<0.01$, or ${ }^{* * *} \mathrm{p}<0.001$, the MPP+-treated group is expressed as ${ }^{\# \# \#} \mathrm{p}<0.001$, and the MPP ${ }^{+}$scrambled-siRNA group is expressed as ${ }^{\$} \mathrm{p}<0.05$.

High Sim levels can increase cellular endogenous ROS levels by regulating ERK1/2

Previous studies revealed that mitogen-activated protein kinases, especially ERK, are important upstream regulators of Nrf2. Thus, we speculated that Sim activates the Nrf2 signaling pathway by regulating ERK1/2. As shown in Fig. 4a, the level of Phospho ERK1/2 in Sim-treated SH-SY5Y cells was significantly increased by $130.1 \pm 6.45 \%\left(^{* *} p<0.01, \mathrm{n}=\right.$ $5)$ compared with that of the control cells. The Nrf2-related anti-oxidant genes were then analyzed.

When SH-SY5Y cells were treated with $0.1,0.5$, or $1.0 \mu \mathrm{M}$ Sim, no significant changes were observed in the MDA (Fig. 4b), GSH (Fig. 4C), and SOD (Fig. 4d) levels. However, the 1.5 $\mu \mathrm{M}$ Sim treatment induced significant changes in the MDA (Fig. 4 b) $\left.{ }^{*} p<0.05, \mathrm{n}=5\right)$ and SOD (Fig. $4 \mathrm{~d}) \mathrm{C}^{*} p<0.05, \mathrm{n}=5$ ) levels. These results suggested that at high concentrations, Sim can increase intracellular ROS levels, which indicates the Sim-induced activation of Nrf2 is due to an increase in ERK1/2. 
Fig. 4. Sim at high concentrations can adjust intracellular ROS levels by regulating ERK1/2. (a) Western blot was used to measure ERK1/2 and Phospho ERK1/2 levels in SHSY5Y cells treated with $1.5 \mu \mathrm{M}$ Sim. (b-d) MDA (b), GSH (c), and SOD (d) levels were measured in SH-SY5Y cells treated with 0.1 , $0.5,1.0$, or $1.5 \mu \mathrm{M}$ Sim. The results of the control group are expressed as ${ }^{*} \mathrm{p}<0.05$ or ${ }^{* *} \mathrm{p}<0.01$.

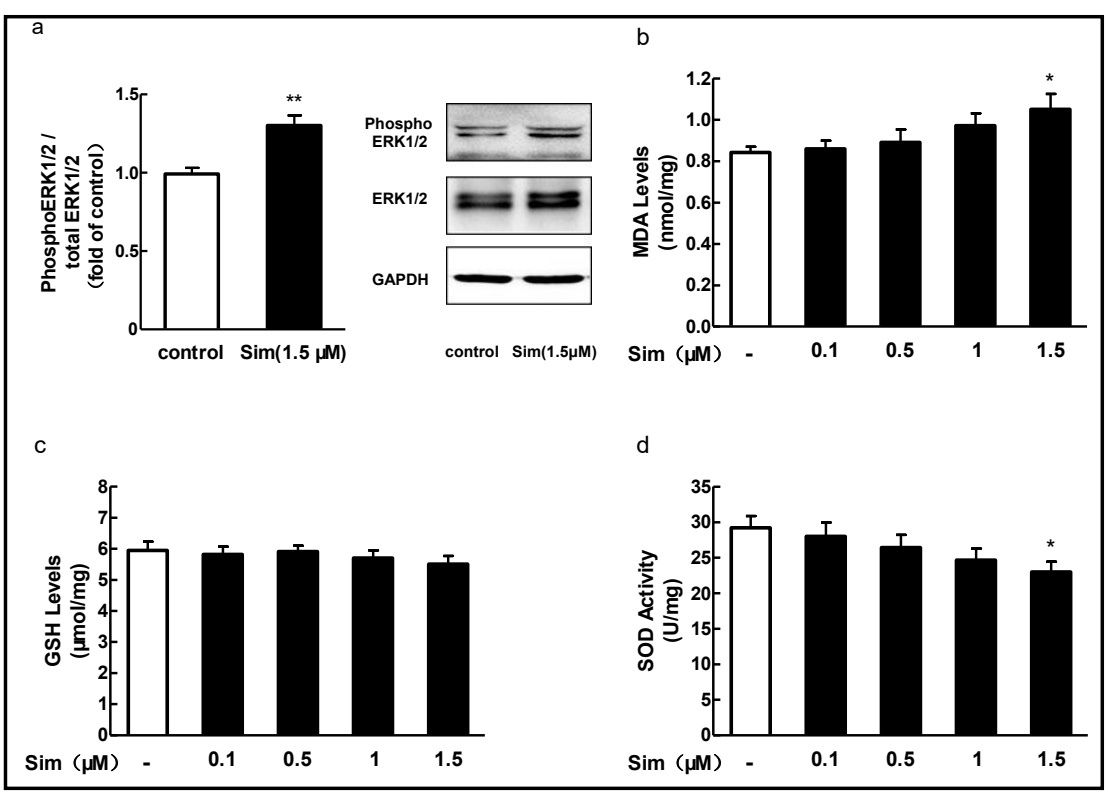

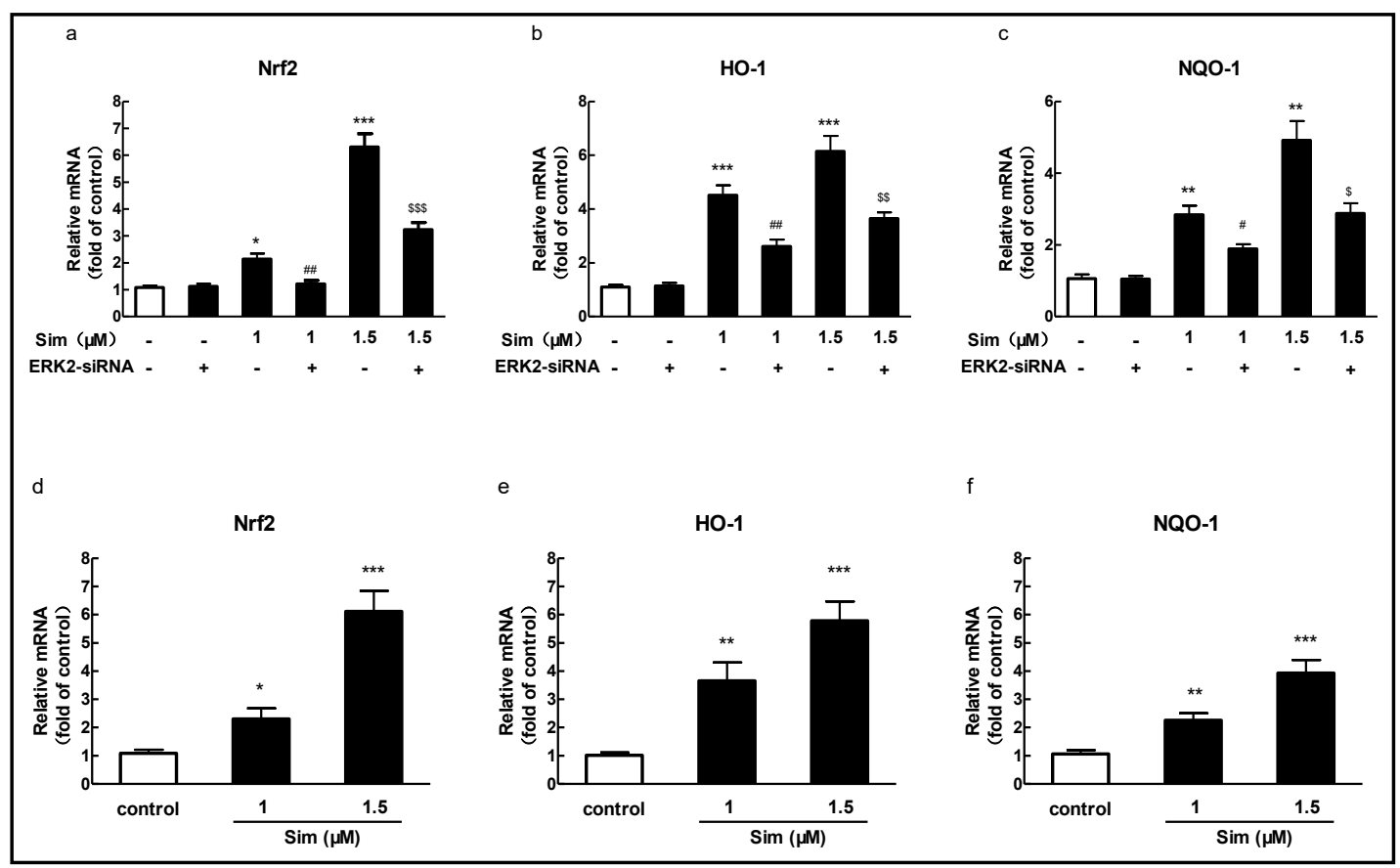

Fig. 5. ERK1/2 siRNA inhibits the Sim-induced upregulated expression of anti-oxidant genes. (a-c) Fluorescent qPCR was used to measure Nrf2 (a), HO-1 (b), and NQO-1 (c) mRNA levels in SH-SY5Y cells treated with 1.0 or $1.5 \mu \mathrm{M}$ Sim, with or without ERK1/2 siRNA. (d-f) Fluorescent qPCR was used to analyze primary cultured substantia nigra neurons treated with 1.0 or $1.5 \mu \mathrm{M}$ Sim regarding Nrf2 (a), HO-1 (b), and NQO-1 (c) mRNA levels. The results of the control group are expressed as ${ }^{*} \mathrm{p}<0.05,{ }^{* *} \mathrm{p}<0.01$, or ${ }^{* * *} \mathrm{p}<0.001$. The results of the $1 \mu \mathrm{M}$ Sim-treated group are expressed as ${ }^{\#} \mathrm{p}<0.05$ or ${ }^{\# \#} \mathrm{p}<0.01$, whereas the results of the $1.5 \mu \mathrm{M}$ Sim-treated group are expressed as ${ }^{\$} \mathrm{p}<0.05,{ }^{\$} \mathrm{p}<0.01$, or ${ }^{\$ \$} \mathrm{p}<0.001$. 
Fig. 6. Sim can inhibit NOX production and activity. (a) Western blot was used to measure the NOX2 level in SH-SY5Y cells treated with $1.5 \mu \mathrm{M}$ Sim and $1 \mathrm{mM} \mathrm{MPP}^{+}$. (b) The Micro NOX assay kit was used to measure NOX activity in SH-SY5Y cells treated with $1.5 \mu \mathrm{M}$ Sim and $1 \mathrm{mM} \mathrm{MPP}^{+}$. The results of the control group are expressed as ${ }^{*} \mathrm{p}<0.05$ or ${ }^{* *} \mathrm{p}<0.01$, while the results of the $\mathrm{MPP}^{+}$-treated group are expressed as ${ }^{\#} \mathrm{p}<0.05$.

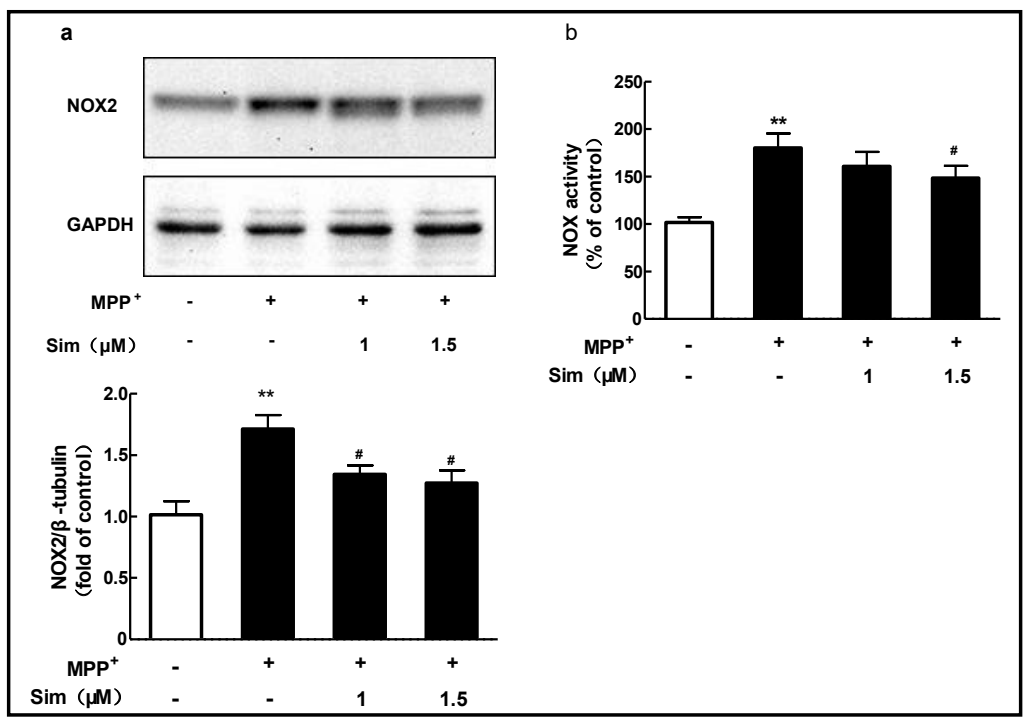

ERK1/2 can significantly inhibit the Sim-induced upregulated expression of Nrf2-related anti-oxidant genes

To further verify the effect of Sim on Nrf2 expression via an ERK1/2-mediated mechanism, we investigated the effect of ERK1/2 siRNA on the activation of Nrf2 expression induced by Sim. After 1 and $1.5 \mu$ M Sim treatments of SH-SY5Y cells, the mRNA levels of Nrf2 (Fig. 5a), HO-1 (Fig. 5b), and NQO-1 (Fig. 5c) were higher than the corresponding levels in the control group $\left({ }^{*} p<0.05,{ }^{* *} p<0.01\right.$, or $\left.{ }^{* * *} p<0.001, \mathrm{n}=5\right)$. Comparable results were also observed in primary cultured dopaminergic neurons (Fig. $5 \mathrm{~d}-\mathrm{f})\left({ }^{*} p<0.05,{ }^{* *} p<0.01\right.$, or ${ }^{* * *} p$ $<0.001, \mathrm{n}=5$ ). However, when SH-SY5Y cells were treated with ERK siRNA and Sim, the Sim-induced upregulated expression of Nrf2 (Fig. 5a) $\left(\mathrm{n}=5\right.$, ${ }^{\# \#} p<0.01 v s .1 \mu \mathrm{M}$ Sim and ${ }^{\$ \$ \$} p$ $<0.001$ vs. $1.5 \mu \mathrm{M}$ Sim), HO-1 (Fig. 5b) ( $=5$, ${ }^{\# \#} p<0.01$ vs. $1 \mu \mathrm{M}$ Sim and ${ }^{\$ \$} p<0.01$ vs. 1.5 $\mu \mathrm{M}$ Sim), and NQO-1 (Fig. 5c) ( $=5,{ }^{\#} p<0.05 v s .1 \mu \mathrm{M}$ Sim and ${ }^{\$} p<0.05 v s .1 .5 \mu \mathrm{M} \mathrm{Sim}$ ) was significantly attenuated. These results indicated that Sim can activate the Nrf2 anti-oxidant system by regulating the ERK pathway when cells are subjected to sustained increases in oxidative stress.

\section{Sim inhibits NOX2 expression}

Previous studies revealed that Sim can inhibit autophagy in muscle cells by inhibiting CYBB/NOX2. Therefore, we explored whether this mechanism is involved in the protective effect of Sim against MPP+-induced oxidative stress and cytotoxicity. Compared with the control group level, the NOX2 level (Fig. 6a) $\left({ }^{* *} p<0.01, \mathrm{n}=5\right)$, and NOX activity (Fig. 6b) $\left(^{* *} p\right.$ $<0.01, \mathrm{n}=5$ ) were significantly increased in MPP+- treated SH-SY5Y cells. Although $1 \mu \mathrm{M} \mathrm{Sim}$ did not affect the MPP'-induced NOX activity (Fig. $6 \mathrm{~b})(\mathrm{n}=5)$, the abundance of NOX2 was significantly increased $v s$. the control group (Fig. 6a) $\left({ }^{\#} p<0.05, \mathrm{n}=5\right)$.

\section{Discussion}

In this study, we analyzed for the first time the effects of Sim in a PD cell model. Sim is a lipid-lowering medication that works by decreasing cholesterol production by the liver. Furthermore, it is used in the treatment of hyperlipidemia and coronary heart disease and to upregulate the expression of genes related to the endogenous anti-oxidant system (Nrf2, HO-1, and NQO-1) in SH-SY5Y cells via an ERK1/2-mediated mechanism. With Sim, this mechanism can decrease the intracellular oxidative stress caused by $\mathrm{MPP}^{+}$in dopaminergic neuronal cells. The data from this study provide new insights into the neuroprotective mechanisms of Sim. 


\section{Cellular Physiology Cell Physiol Biochem 2018;51:1957-1968

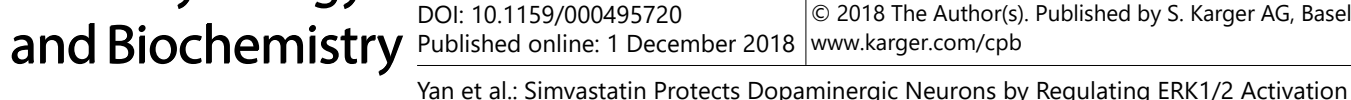 \\ of Anti-Oxidants}

Previous clinical studies revealed that Sim has the potential to decrease the risk of PD by lowering oxidative stress $[12,13]$, and it can also affect the suppressive potential of pathological alpha-Syn by lowering cholesterol levels [14]. The fact that Sim can cross the blood-brain barrier as well as the considerable evidence supporting the beneficial effects of Sim on the central nervous system make Sim one of the more promising drugs for treating neurodegenerative diseases, including stroke $[15,16]$ and PD $[17,18]$. Earlier investigations confirmed that ROS is produced during the maintenance of an essential metabolic balance in the body, as a series of anti-oxidant mechanisms stabilize the redox balance of cells $[19,20]$. Homeostatic changes induce excessive ROS production, leading to DNA mutations and protein dysfunction that eventually result in damaged dopaminergic neurons and neuropathology. We used SH-SY5Y neurons, which have dopaminergic neuronal properties and are positive for tyrosine hydroxylase. We observed that Sim scavenges ROS, and a pre-incubation with Sim can significantly alleviate the oxidative stress of SH-SY5Y cells. Moreover, Sim is also beneficial because it protects dopaminergic neurons against PD lesions.

A series of studies concluded that Sim exhibits anti-oxidant activity [21] in different cells, including nerve cells. However, to our knowledge, the mechanism underlying the antioxidant system has not been elucidated. Our previous study indicated that Sim can regulate NMDA receptors and exhibit anti-anxiety and anti-inflammatory activities in a PD cell model [22]. In this study, we used a modeling approach different from that used in previous studies to evaluate the effect of Sim on MPP'-induced oxidative stress in PD models.

Interestingly, in this study, we observed the protective effect of Sim against oxidative stress in neurons. Meanwhile, we determined that as the Sim dose increases, the level of ROS declines in the $\mathrm{MPP}^{+}$cell model, implying that Sim can trigger the resistance of nerve cells against $\mathrm{MPP}^{+}$oxidative stress. We further observed the effect of Sim on NOX2 production and activity in cells affected by MPP+-induced oxidative stress. Sim can inhibit NOX2 production in neurons with a dose range consistent with that reported in a previous study involving muscle cells [23]. Moreover, we observed NOX activity decreased after the Sim treatment, and was negatively correlated with Sim dose. Therefore, the effect of Sim on oxidative stress may be attributed to the inhibition of NOX activity.

Additionally, we observed that Sim had a direct effect on the removal of exogenous ROS. As previously demonstrated, Sim can activate Nrf2 in human melanoma cells and colon cancer cells [24]. Another study confirmed that Nrf2 is an important transcription factor involved in the anti-oxidant response [25] of cells, during which it binds to the anti-oxidant genes, $H O-1$ and $N Q O-1$ [26]. Moreover, we found that Sim was able to upregulate ERK1/2 expression.

Furthermore, the activation of ROS/ERK/Nrf2 signaling pathways may improve the anti-oxidative stress and anti-inflammatory properties of Sim. Therefore, we believe that the results of this study highlight the Sim-induced decrease in the oxidative stress of $\mathrm{MPP}^{+} \mathrm{PD}$ model cells. Regarding the upregulated $\mathrm{Nrf2}, \mathrm{HO}-1$, and $\mathrm{NQO}-1$ expression levels, we believe that statins, which are HMG-CoA reductase inhibitors, function by blocking hydroxymethyl acid metabolic pathways. Furthermore, the Sim-induced high Nrf2, HO-1, and NQO-1 expression levels decreased after ERK1/2 expression was silenced via ERK1/2 siRNA. Thus, we conclude that $\mathrm{MPP}^{+}$blocks the respiratory chain that causes elevated intracellular ROS levels. Sim increased ERK expression and activated the expression of Nrf2 and the associated anti-oxidant genes, thereby increasing resistance to oxidative stress.

It is noteworthy that in the cell anti-oxidant experiments, we only used the $\mathrm{MPP}^{+}$induced PD cell model and did not observe other drug-induced oxidative stresses. However, Sim was able to increase ERK1/2 expression levels as well as the expression of Nrf2 and related anti-oxidant genes, which was necessary to verify our research in vivo. Our future research will lean toward the same direction, and we believe that other oxidative stress models will display comparable effects. 


\section{Cellular Physiology Cell Physiol Biochem 2018;51:1957-1968 \begin{tabular}{ll|l} 
DOI: 10.1159/000495720 & $\begin{array}{l}\text { O } 2018 \text { The Author(s). Published by S. Karger AG, Basel } \\
\text { www.karger.com/cpb }\end{array}$ \\
\hline
\end{tabular} \\ Yan et al.: Simvastatin Protects Dopaminergic Neurons by Regulating ERK1/2 Activation of Anti-Oxidants}

We previously demonstrated that Sim can enhance the resistance to inflammation by regulating NMDA in a 6-OHDA PD mouse model. In this study, we revealed that Sim can regulate ERK in a cell model by activating Nrf2, which enhances the resistance to neuronal oxidative stress. Therefore, we believe that Sim is able to significantly decrease the onset of PD largely because of its anti-inflammatory effect against oxidative stress. However, further research is necessary to prove the protective effect of Sim on dopaminergic neurons.

\section{Conclusion}

In conclusion, this study shows that Sim regulates ERK1/2 expression and activates Nrf2 and associated anti-oxidant systems. Thus, Sim helps alleviate cardiovascular diseases by combating the oxidative stress caused by $\mathrm{MPP}^{+}$, and is expected to have a strong protective effect against neurodegenerative diseases.

\section{Acknowledgements}

This study was supported by the National Natural Science Fund (Grant No. U1304809).

\section{Disclosure Statement}

The authors declare no competing financial interests.

\section{References}

1 Hurtig H, Trojanowski J, J, Ewbank D, Schmidt M, Lee V, Clark C, Glosser G, Stern M, Gollomp S, Arnold S: Alpha-synuclein cortical Lewy bodies correlate with dementia in Parkinson's disease. Neurology 2000;54:1916-1921.

-2 Zhou C, Huang Y, Przedborski S: Oxidative Stress in Parkinson's Disease. Annals of the New York Academy of Sciences 2008;1147:93-104.

-3 Dauer W, Przedborski S: Parkinson's Disease : Mechanisms and Models. Neuron 2003;39:889.

4 Höglinger GU, Carrard G, Michel PP, Medja F, Lombès A, Ruberg M, Friguet B, Hirsch EC: Dysfunction of mitochondrial complex I and the proteasome: interactions between two biochemical deficits in a cellular model of Parkinson's disease. Journal of neurochemistry 2003;86:1297-1307.

5 Mcguinness B, Craig D, Bullock R, Passmore P: Statins for the prevention of dementia. Cochrane Database Syst Rev 2016;1:CD003160.

6 Huang X, Alonso A, Guo X, Umbach DM, Lichtenstein ML, Ballantyne CM, Mailman RB, Mosley TH, Chen $\mathrm{H}$ : Statins, plasma cholesterol, and risk of Parkinson's disease: A prospective study. Movement Disorders Official Journal of the Movement Disorder Society 2015;30:552-559.

7 Geifman N, Brinton RD, Kennedy RE, Schneider LS, Butte AJ: Evidence for benefit of statins to modify cognitive decline and risk in Alzheimer's disease. Alzheimers Research \& Therapy 2017;9:10.

-8 Yan J, Sun J, Huang L, Fu Q, Du G: Simvastatin prevents neuroinflammation by inhibiting N-methyl-Daspartic acid receptor 1 in 6-hydroxydopamine-treated PC12 cells. Journal of Neuroscience Research 2014;92:634-640.

-9 Xu YQ, Long L, Yan JQ Wei L, Pan MQ, Gao HM, Zhou P, Liu M, Zhu CS, Tang BS: Simvastatin induces neuroprotection in 6-OHDA-lesioned PC12 via the PI3K/AKT/caspase 3 pathway and anti-inflammatory responses. Cns Neuroscience \& Therapeutics 2013;19:170.

10 Chang Y, Li S, Guo W, Yang Y, Zhang W, Zhang Q He Y, Yi X, Cui T, An Y: Simvastatin Protects Human Melanocytes from H2O2-Induced Oxidative Stress by Activating Nrf2. Journal of Investigative Dermatology 2017;137:1286. 


\section{Cellular Physiology Cell Physiol Biochem 2018:51:1957-1968

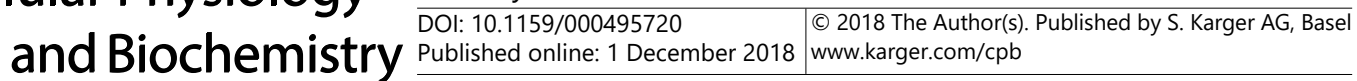 \\ Yan et al.: Simvastatin Protects Dopaminergic Neurons by Regulating ERK1/2 Activation \\ of Anti-Oxidants}

11 Hua F, Shu Q, Guan X, Zhao J, Yan J, Li X, Liu J, Jia Z, Jian S, Li J: Sinomenine Protects PC12 Neuronal Cells against H 2 O 2 -induced Cytotoxicity and Oxidative Stress via a ROS-dependent Up-regulation of Endogenous Antioxidant System. Cellular \& Molecular Neurobiology 2017;1-12.

12 Sohn HM, Hwang JY, Ryu JH, Kim J, Park S, Park J, Han SH: Simvastatin protects ischemic spinal cord injury from cell death and cytotoxicity through decreasing oxidative stress: in vitro primary cultured rat spinal cord model under oxygen and glucose deprivation-reoxygenation conditions. Journal of Orthopaedic Surgery \& Research 2017;12:36.

13 Baror D, Baror R, Rael LT, Brody EN: Oxidative stress in severe acute illness. Redox Biology 2015;4:340-345.

14 Carroll CB, Rkh W: Simvastatin as a Potential Disease-Modifying Therapy for Patients with Parkinson's Disease: Rationale forÂ Clinical Trial, and Current Progress. J Parkinsons Dis 2017;1-24.

15 Zhu MX, Lu C, Xia CM, Qiao ZW, Zhu DN: Simvastatin pretreatment protects cerebrum from neuronal injury by decreasing the expressions of phosphor-CaMK II and AQP4 in ischemic stroke rats. Journal of Molecular Neuroscience 2014;54:591-601.

16 Reuter B, Rodemer C, Grudzenski S, Meairs S, Bugert P, Hennerici MG, Fatar M: Effect of Simvastatin on MMPs and TIMPs in Human Brain Endothelial Cells and Experimental Stroke. Translational Stroke Research 2015;6:156.

17 Brakedal B, Haugarvoll K, Tzoulis C: Simvastatin is associated with decreased risk of Parkinson disease. Annals of Neurology 2016;81:329-330.

18 Lee YC, Lin CH, Wu RM, Lin MS, Lin JW, Chang CH, Lai MS: Discontinuation of statin therapy associates with Parkinson disease: a population-based study. Neurology 2013;81:410.

19 Zuo L, Motherwell MS: The impact of reactive oxygen species and genetic mitochondrial mutations in Parkinson's disease. Gene 2013;532:18-23.

20 Hwang 0: Role of Oxidative Stress in Parkinson's Disease. Experimental Neurobiology 2013;22:11.

21 Davignon J, Mason RP: The antioxidant effects of statins. Coronary Artery Disease 2004;15:251.

22 Yan JQ, Ma YJ, Sun JC, Bai SF, Huang LN: Neuroprotective effect of lovastatin by inhibiting NMDA receptor1 in 6-hydroxydopamine treated PC12 cells. International Journal of Clinical \& Experimental Medicine 2014;7:3313-3319.

23 Whitehead NP: Enhanced autophagy as a potential mechanism for the improved physiological function by simvastatin in muscular dystrophy. Autophagy 2016;12:705.

24 Jang HJ, Hong EM, Kim M, Kim JH, Jang J, Park SW, Byun HW, Koh DH, Choi MH, Kae SH: Simvastatin induces heme oxygenase-1 via NF-E2-related factor 2 (Nrf2) activation through ERK and PI3K/Akt pathway in colon cancer. Oncotarget 2016;7:46219.

25 Nguyen T, Nioi P, Pickett CB: The Nrf2-antioxidant response element signaling pathway and its activation by oxidative stress. Journal of Biological Chemistry 2009;284:13291-13295.

26 Kim HJ, Min Z, Kim SK, Cho JJ, Chang HS, Joe Y, Chung HT: CO/HO-1 Induces NQO-1 Expression via Nrf2 Activation. Immune Netw 2011;11:376-382. 